\title{
Tourismusbedingte Landschaftsveränderungen im Urteil der Touristen
}

\author{
Wie wichtig ist die landschaftliche Schönheit \\ für den Tourismus im Berggebiet? Die Ausgangslage
}

Die MaB-Studien' haben es gezeigt: Der Tourismus ist in vielen Berggemeinden wichtigster und unersetzlicher Wirtschaftsfaktor (KRIPPENDORF 1986b). Es liegt daher auf der Hand, daß die langfristige Stabilität solcher lokaler Wirtschaftssysteme in starkem Maße von der Erhaltung der bedeutendsten Angebotsfaktoren im Bergtourismus abhängt; insbesondere von der landschaftlichen Schönheit, denn sie ist laut verschiedensten Gästebefragungen eines der wichtigsten Motive für die Wahl eines Ferienortes (KRIPPENDORF 1986a; WIESMANN 1986).

$\mathrm{Daß}$ die Landschaft trotz dem ausgewiesenen Bedürfnis nach ihrer Intaktheit in den letzten Jahrzehnten starken Veränderungen unterlag, gründet im ebenfalls vorhandenen Gästebedürfnis nach einer Infrastruktur, die der Mehrheit der Touristen das Landschaftserlebnis überhaupt erst ermöglicht. Aus diesem zweiten grundlegenden Gästebedürfnis läßt sich im Gegensatz zu jenem nach einer intakten Landschaft kurzfristig ein ökonomischer Nutzen ziehen, indem Produkte wie Unterkünfte, Beförderung usw. verkauft werden. Die damit verbundenen Landschaftsveränderungen akzeptierte die Mehrheit der Touristen, solange das Infrastrukturangebot knapp und gleichzeitig die intakte Landschaft in genügendem Maße vorhanden war (TSCHURTSCHENTHALER 1986).

In jüngster Zeit ist aber die komfortable Situation des Nachfrageüberhanges ins Wanken geraten: Seit Beginn der achtziger Jahre stagniert im Schweizer Bergtourismus die Nachfrage, während das Angebot gleichzeitig weiter ausgebaut wurde (FISCHER 1989; MESSERLI 1989). Wegen der damit entstandenen Käufermarktverhältnisse müssen sich die einzelnen Tourismusanbieter wieder vermehrt nach den Bedürfnissen der Nachfrager richten und versuchen, sich mittels des Preis-Leistungs-Verhältnisses der Angebote gegenüber der Konkurrenz zu profilieren. Für den Schweizer Tourismus steht dabei wegen seines hohen Preisniveaus die Leistung im Vordergrund: Sein Angebot muß sich besonders im Hinblick auf die mit der Konstitution des EG-Binnenmarktes sich weiter verschärfenden Konkurrenzverhältnisse durch Individualität und hochstehende Qualität ausweisen, damit die Nachfrage erhalten oder gar gesteigert werden kann (KELLER 1988). Und weil das Infrastrukturangebot zwischen den verschiedenen Tourismusregionen immer vergleichbarer wird (MAGGI 1983), dürften künftig ursprüng- liche Angebote wie die landschaftliche Schönheit für die Profilierung im Vordergrund stehen.

Mit großer Wahrscheinlichkeit wird sich also die Landschaftsqualität zu einem ausschlaggebenden Wettbewerbsfaktor entwickeln. Diesem Risiko - oder dieser Chance! - sollte durch entsprechende Ferienortsplanungen Rechnung getragen werden. Dazu muß bekannt sein, welche Landschaft den Touristen am besten gefällt sowie welche Veränderungen von ihnen negativ erlebt werden und damit das Risiko ihres Fernbleibens erhöhen. Das Ziel der durchgeführten Studie war es, zur Erarbeitung der dazu notwendigen Grundlagen beizutragen.

\section{Welche Landschaft gefällt den Touristen? Die Hypothese}

Laut den theoretischen Ausführungen und empirischen Untersuchungen von NOHL \& NEUMANN (1986) gefällt eine Landschaft den meisten Betrachtern aufgrund ihrer Vielfalt, Struktur, Naturnähe und Eigenart ${ }^{2}$. Eine optimale Zusammensetzung dieser Eigenschaften weist im Berggebiet die traditionelle bäuerliche Kulturlandschaft auf: Die darin deutlich erkennbaren Spuren menschlicher Naturbearbeitung geben ihr eine Struktur, in der sich der Betrachter orientieren kann. Die Art und Weise der Naturbearbeitung wurde - im Rahmen der naturräumlichen Voraussetzungen - weitgehend von der Bevölkerung bestimmt und unterscheidet sich zwischen verschiedenen Regionen. Die Spuren der Naturbearbeitung bringen daher regionale Identität zum Ausdruck (ABT 1983) und verleihen der traditionellen Kulturlandschaft ihre Eigenart. Ihre Naturnähe und Vielfalt gründet in der mangels verfügbarer Fremdenergie notwendigen Anpassung der Bewirtschaftung an die Gegebenheiten des Naturraums (BÄTZING 1988).

Durch die tourismusbedingten Veränderungen der Landschaft werden deren erwünschten Eigenschaften beeinträchtigt: Naturnähe geht der Landschaft verloren, weil - unter Einsatz von Fremdenergie und moderner In-

Marcel Hunziker, Lic. phil. Geograph, Eidg. Forschungsanstalt für Wald, Schnee und Landschaft, $\mathrm{CH}-8903$ Birmensdorf. 
genieurtechnik - zur Befriedigung touristischer Infrastrukturbedürfnisse Bauten und Anlagen entstehen, die sich über die naturräumlichen Gegebenheiten hinwegzusetzen suchen. Mit der Verdrängung des Naturaspektes aus der Kulturlandschaft wird auch deren Vielfalt vermindert. Die Eigenart der Landschaft wird gefährdet, weil die touristische Entwicklung die Landschaft zu vereinheitlichen droht; beispielsweise durch den verbreiteten "Chaletstil». Die boomartige Expansion der touristischen Siedlungen und Transportanlagen verändert die ausgewogene traditionelle Struktur. Häufig entstehen dabei unübersichtliche Anhäufungen von Landschaftselementen oder - wo dem mit planerischen Maßnahmen entgegengewirkt wird - künstlich wirkende Reißbrettstrukturen.

Als Hypothese, die es in der Studie zu überprüfen galt, wurde deshalb angenommen, daß die traditionelle bäuerliche Kulturlandschaft den Berggebietstouristen am besten gefällt und daß die tourismusbedingten Abweichungen von ihr einen ästhetischen Verlust bedeuten.

\section{Wie wird die ästhetische Beurteilung von Landschaften «gemessen»? Die Methode}

Die Hypothese wurde mittels Fototests in einer mündlichen Befragung von 204 zufällig ausgewählten Gästen Grindelwalds überprüft. Dabei wurden nur Beurteilungen von Landschaftsveränderungen untersucht, welche hauptsächlich vom Tourismus verursacht und prinzipiell durch planerische Maßnahmen beeinflußbar sind, nämlich die bauliche Entwicklung von Streusiedlungen und Haufendörfern sowie die Erstellung von Straßen und touristischen Transportanlagen. Für jeden dieser vier Veränderungstypen wurde die zunehmende Abweichung von der traditionellen Kulturlandschaft in einer Serie von fünf Fotos dargestellt (Abb. 1).

Die Befragten erhielten die erwähnten 20 Serienbilder gemischt mit zehn weiteren Fotos. Letztere dienten der Ablenkung von den Serienbildern und wurde nicht in die Auswertungen einbezogen. Für die Erfassung der ästhetischen Urteile wurde in einem ersten Schritt das in der Psychologie bewährte "Q-Sort-Verfahren" (STEPHENSON 1953) angewendet (Abb. 2): Die Befragten mußten jeden abgebildeten Landschaftsausschnitt einer von sieben Gefallensstufen zuordnen, wobei die Anzahl Bilder je Stufe (in der Form einer Normalvorteilung) vorgeschrieben war. Mit diesem Vorgehen wurde ein (quasi)metrisches Skalenniveau erzeugt, was Mittelwertsberechnungen und -vergleiche ermöglichte. So konnte der "Schönheitswert» einer dargestellten Landschaft als durchschnittliche Einstufung des Fotos berechnet werden.

In einem zweiten Schritt bewerteten die Befragten die Fotos nochmals, indem sie für jedes Bild zu entscheiden hatten, ob sie die darauf abgebildete Landschaft schön finden oder nicht. Damit konnte für jeden Zustand der Landschaftsveränderung festgestellt werden, wie vielen Touristen dieser prinzipiell noch gefällt.
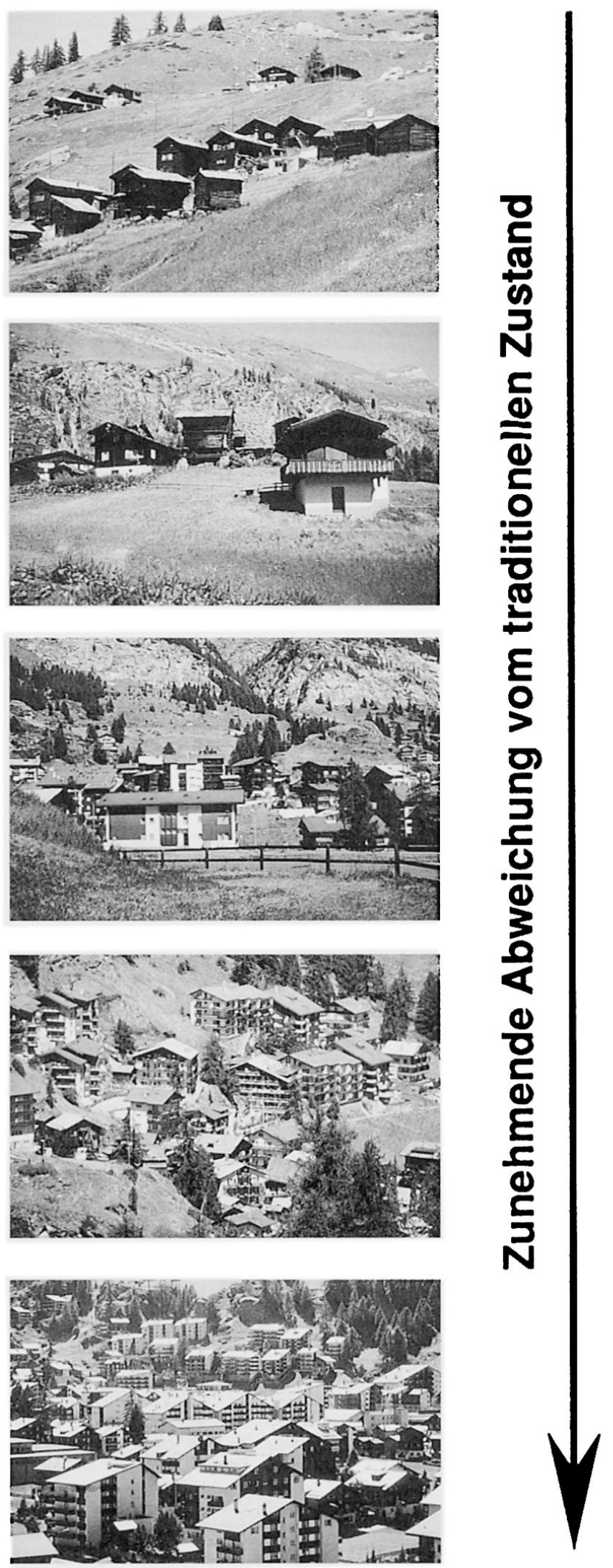

Abb.1 Beispiel einer Fotoserie, die eine zunehmende Abweichung von einem traditionellen Haufendorf darstellt. 


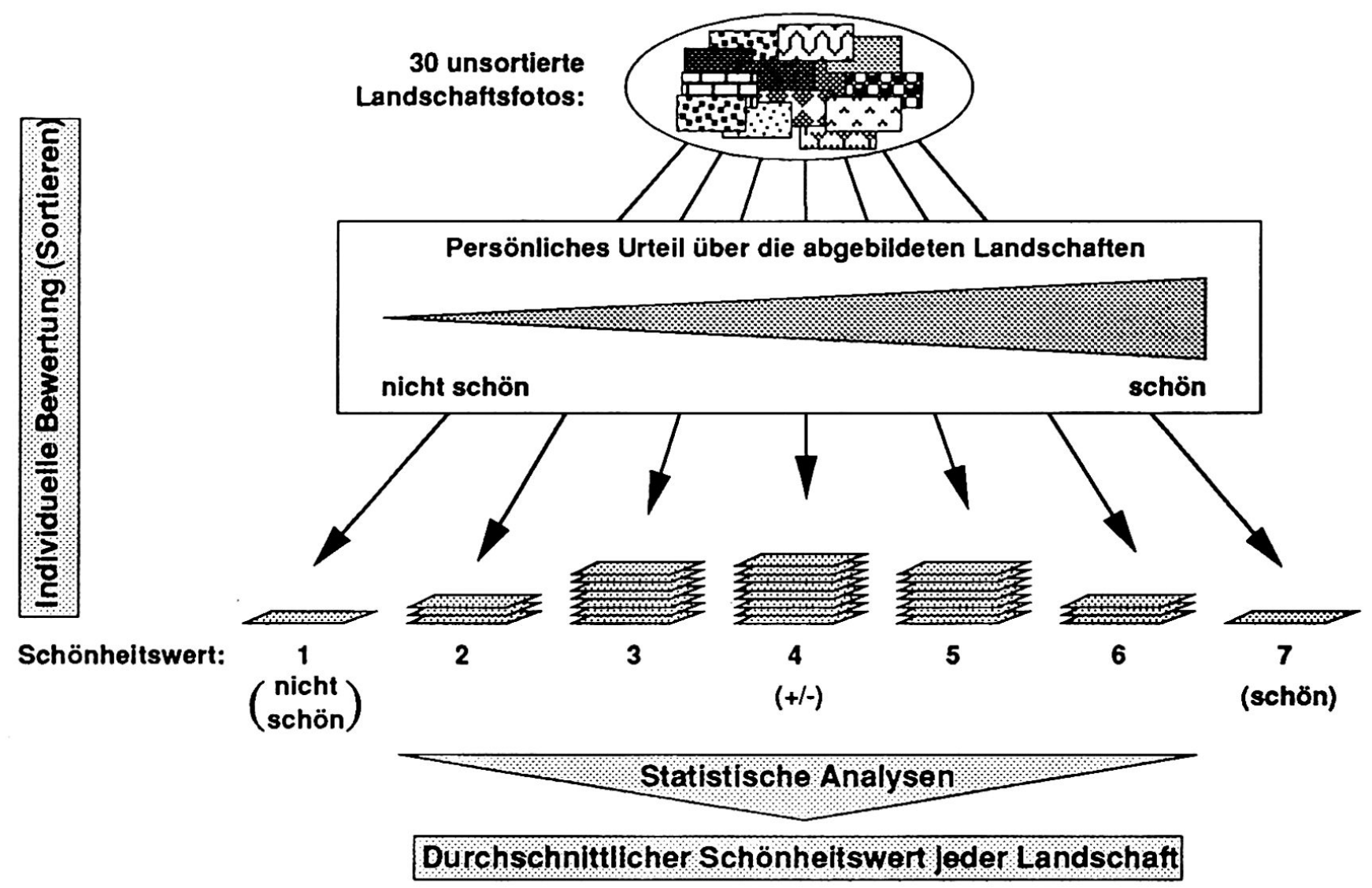

Abb. 2 Schematische Darstellung des Verfahrens zur Erhebung der ästhetischen Urteile über Landschaften (Q-Sort-Verfahren).

Ist die traditionelle Kulturlandschaft ideal? Das Hauptergebnis

In der Abb. 3 sind die Schönheitswerte der 20 Serienfotos dargestellt. Für die Überprüfung der Hypothese war in erster Linie von Interesse, ob die Schönheitswerte mit zunehmender Abweichung vom traditionellen Zustand geringer werden. Weil die Existenz dieses Zusammenhangs nicht alleine aufgrund der deskriptiven Statistik beurteilt werden konnte, wurde als Entscheidungshilfe ein nonparametrischer Trendtest eingesetzt.

Bei der Haufendorfserie wird schon aus der Graphik ersichtlich, daß die Entwicklung vom traditionellen $\mathrm{Zu}$ stand hin zum modernen, beinahe städtischen Siedlungsgebilde (Abb.1) - charakterisiert durch die Verwendung ortsfremder Baumaterialien sowie der touristischen Nutzung entsprechende Gebäudedimensionen und -proportionen - einen ästhetischen Verlust bedeutet. Dieser Zusammenhang ist im Trendtest auf dem 5\%-Niveau signifikant, selbst wenn das zweite Bild der Haufendorfserie etwas höher bewertet wurde als das erste. Die durchschnittlich hohe Einstufung der wegen eines vorhandenen Ferienchalets leicht vom traditionellen $\mathrm{Zu}$ stand abweichenden Siedlung (2. Bild in Abb.1) zeigt auf, daß die Touristen geringe Landschaftsveränderun- gen entsprechend ihren Nutzungsbedürfnissen tolerieren.

Die Entwicklung von der traditionellen Streusiedlung hin zur verdichteten und schachbrettartig angeordneten Ferienchaletüberbauung scheint die Touristen nicht groß zu stören: Der traditionelle Siedlungszustand unterscheidet sich im Schönheitsurteil kaum von den veränderten, wie schon die Graphik deutlich macht. Auch aufgrund des Trendtestes kann die leichte Abnahme der Schönheitswerte nicht als Folge der zunehmenden Abweichung von der traditionellen Streusiedlung betrachtet werden. Eine Erklärung dieses Ergebnisses mag einerseits darin liegen, daß die Touristen ihr Augenmerk hauptsächlich auf Einzelobjekte und weniger auf die Struktur der Landschaft richteten. Deshalb führte die Streusiedlungsentwicklung mit ihren dominanten Veränderungsmerkmalen - die strukturelle Verdichtung und Geometrisierung - nicht zu entsprechenden ästhetischen Verlusten. Andererseits wurden von den Befragten häufig die raumplanerische Forderung nach sparsamem Umgang mit dem Boden als Legitimation für verdichtetes Bauen angeführt. Hier zeigt sich erneut, was schon aus anderen Studien bekannt ist (NOHL 1990): Die ästhetische Beurteilung von Landschaften ist keine absolut subjektive und rein emotionale Angelegenheit, sondern 


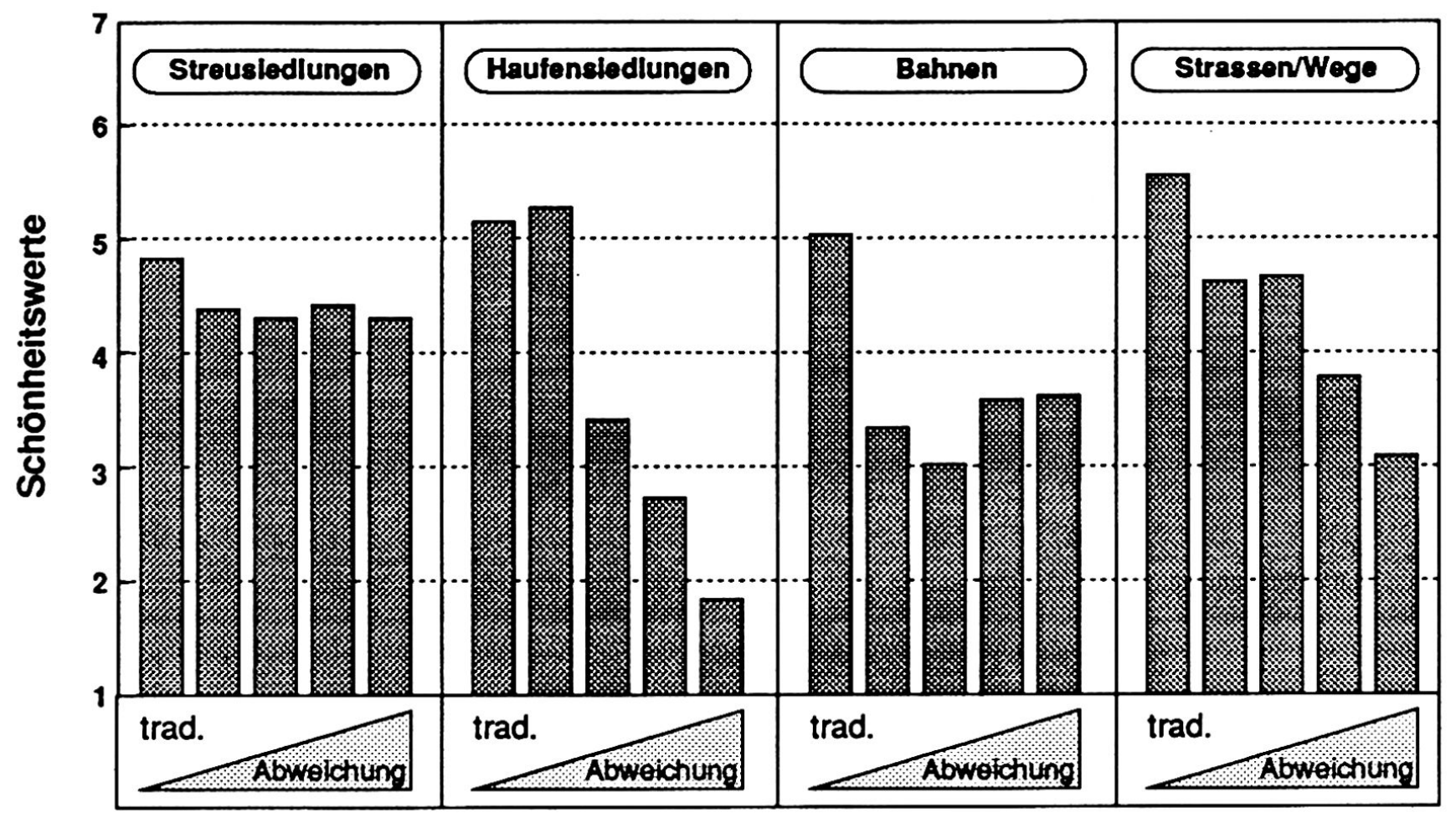

Fotoserien

Abb. 3 Die mit dem Q-Sort-Verfahren empirisch ermittelten "Schönheitswerte" der einzelnen Landschaften. Das erste Foto einer Serie repräsentiert jeweils den traditionellen, das letzte den am stärksten veränderten Zustand.

entspricht durchaus gewissen sozialen Normen und wird von zweckrationalem Denken beeinflußt.

Bei der Beurteilung touristischer Transportanlagen wurde nur der wirklich traditionelle Zustand ohne jegliche touristische Transportanlage (1. Bild der Bahnenserie) hoch bewertet. Hingegen wurden sämtliche Bahnen negativ beurteilt, ohne daß nach der Art der Anlage differenziert wurde (Abb. 3 und 4): Skilifte (2. Bild) stießen genauso auf Mißfallen wie Großkabinenbahnen (5. Bild). Entsprechend liegt denn auch kein signifikanter Zusammenhang zwischen der zunehmenden Abweichung von der traditionellen Kulturlandschaft - charakterisiert durch immer höhere Transportkapazität sowie geringere Geländeanpassung - und dem Schönheitsurteil vor. Der große Bewertungsunterschied zwischen dem traditionellen Zustand ohne und den Landschaften mit irgendeiner Bahn macht aber deutlich, daß die Erstellung von touristischen Transportanlagen unabhängig von ihrer Erscheinung einen erheblichen ästhetischen Verlust bedeutet. Daß die Gondelbahn (4. Bild) und die Großkabinenbahn gar höher bewertet wurden als der Skilift und der Sessellift (3. Bild), mag mit der sommerlichen Benutzbarkeit von ersteren und deren Übereinstimmung mit dem Stereotyp «Schweizer Berge» zusammenhängen: Insbesondere Ausländer hielten die Erscheinung einer roten Bahn in grünen Wiesen für ein besonders typisches Element dieser Landschaft.

Auch der Ausbau des Straßennetzes beeinträchtigt das Landschaftserlebnis, wie die graphische Interpretation nahelegt und der Trendtest mit einer Irrtumswahrscheinlichkeit von höchstens $5 \%$ versichert. Dabei ist das wichtigste Kriterium für die Beurteilung von Straßen nicht etwa deren Belagsart oder Breite, sondern ihre Funktion. Straßen, die zur Hauptsache der Landwirtschaft dienen (1. bis 3. Bild der Straßenserie), gefallen weit besser als reine Verbindungsstraßen (4. und 5. Bild). Hingegen werden Flurstraßen mit unterschiedlichem Belag (2. Bild: Kies; 3. Bild: Asphalt) ungefähr gleich bewertet.

Die Hypothese, den Touristen gefalle die traditionelle Kulturlandschaft am besten, während tourismusbedingte Abweichungen davon als ästhetischer Verlust empfunden werden, ist also bei drei von vier Veränderungstypen nicht zu widerlegen. Vom Tourismus ausgelöste Landschaftsveränderungen können daher - vorläufig und aus der Sicht der Gäste! - in den meisten Fällen als ästhetische Beeinträchtigungen angesehen werden.

\section{Erhöht sich künftig die Sensibilität auf Landschaftsveränderungen? Ein Beispiel eines gruppenspezifischen Ergebnisses}

Die Beurteilungen wurden auch bezüglich Unterschieden zwischen sozialen Gruppen wie beispielsweise verschiedenen Altersgruppen untersucht. Dabei wurden die gruppenspezifischen Schönheitswerte jeder dargestell- 

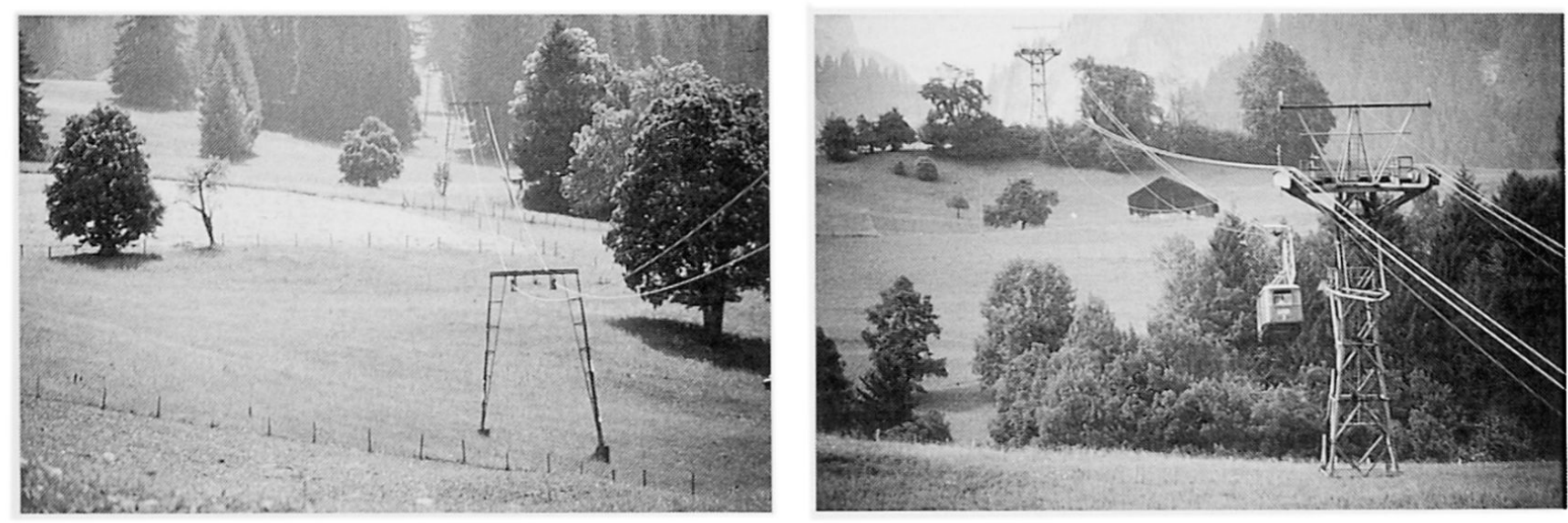

Abb. 4 Ein Ergebnis aus der zweistufigen Fotobeurteilung nach "Schön" - "nicht schön": Ob Skilift oder Großkabinenbahn, touristische Transportanlagen bedeuten immer eine starke Beeinträchtigung der landschaftlichen Schönheit. Den Skilift (2. Foto der Bahnenserie) fanden nur $23 \%$ der Befragten schön, die Großkabinenbahn (5. Foto) nur $29 \%$.

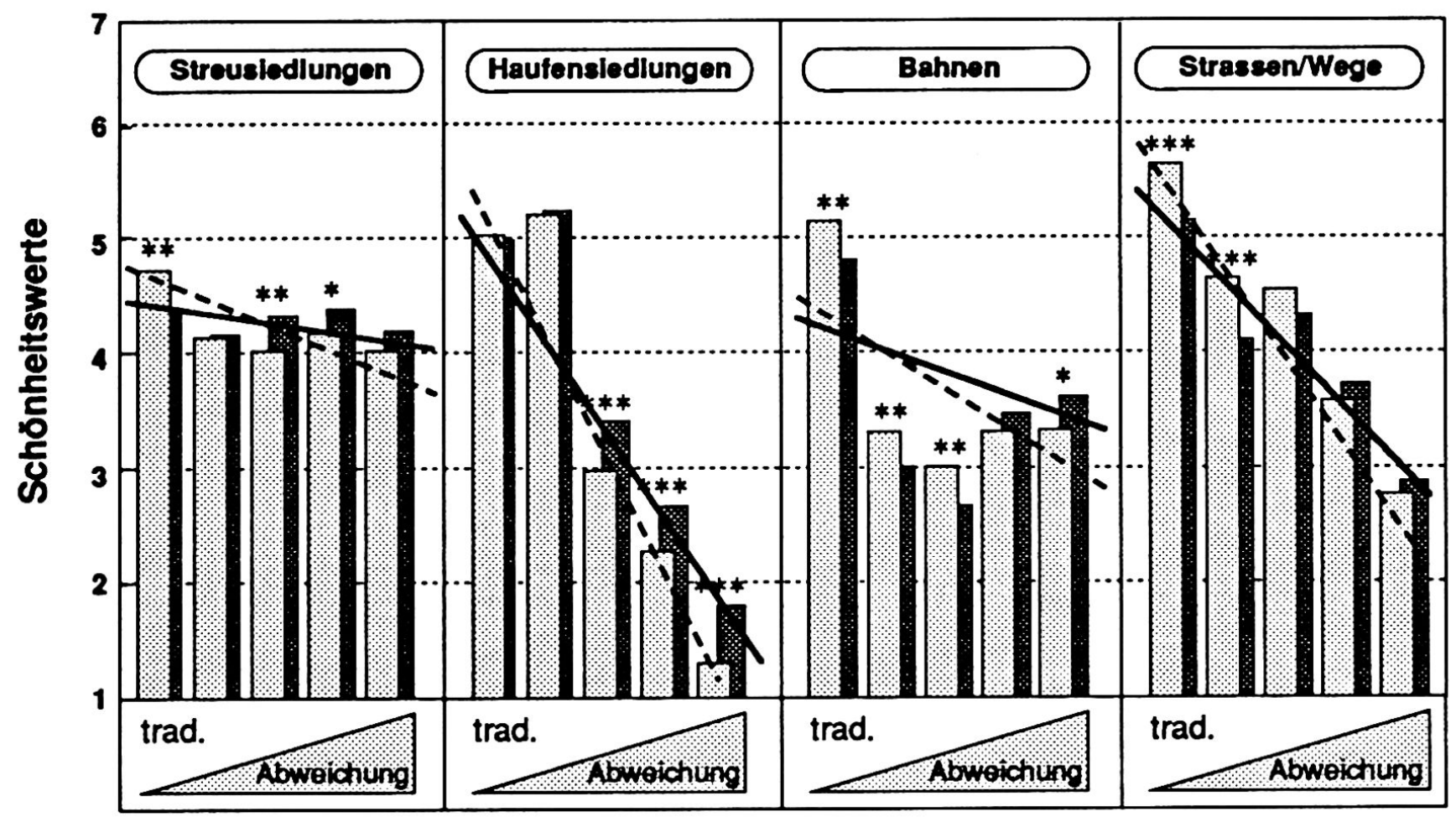

Fotoserien
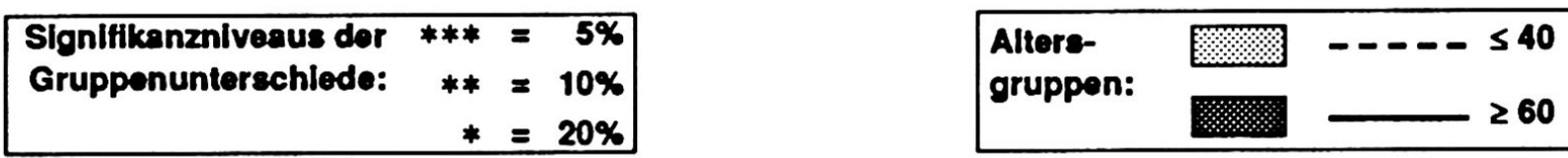

Abb. 5 Die Schönheitswerte der einzelnen Landschaften differenziert nach Altersgruppen. 
ten Landschaft verglichen und die Signifikanz der Unterschiede überprüft. Die Analyse der altersspezifischen Beurteilungen zeigte, daß die jüngeren Gäste die traditionellen Landschaften höher bewerten als die älteren (Abb. 5). Die stark vom traditionellen Zustand abweichenden Landschaften werden hingegen von den Jüngeren negativer bewertet als von den Älteren. Die Jüngeren sind also auf den Prozeß der Abweichung von der traditionellen Kulturlandschaft sensibler als die Älteren. Die in der Abb. 5 eingezeichneten Trendgeraden veranschaulichen diesen Unterschied: Die Steigungen der Geraden - als Indikator für die Sensibilität auf tourismusbedingte Landschaftsveränderungen betrachtet - sind bei den Jüngeren höher als bei den Älteren. In Anlehnung an die einschlägige Literatur ist dieser Sensibilitätsunterschied eher dem allgemeinen Wertewandel als einer altersbedingten Abnahme der individuellen Landschaftssensibilität zuzuschreiben (NOHL \& NEUMANN 1986; ROMEISSSTRACKE 1987). Diesem Sachverhalt muß besondere Beachtung geschenkt werden, da die heutigen Jungen die kaufkräftigen Touristen von morgen, die 40- bis 60 jährigen der Gegenwart die aktiven Senioren der Zukunft sein werden: Die Vertreter der relevanten Nachfragesegmente dürften somit gegenüber Landschaftsveränderungen künftig sensibler sein, womit sich das Risiko des landschaftsbedingten Fernbleibens von Touristen in Zukunft verschärfen könnte.

\section{Gefährden Landschaftsveränderungen \\ die touristische Entwicklung? \\ Die Analyse der Verhaltensrelevanz}

Die Studie gibt auch Hinweise auf erste Nachfragereaktionen: Beispielsweise zeigen die im städtisch anmutenden Zentrum Grindelwalds einquartierten Gäste eine tendenziell geringere Sensibilität auf Abweichungen von der traditionellen Kulturlandschaft als jene mit Unterkunft in der Umgebung Grindelwalds, die noch weitgehend einen traditionellen Charakter aufweist (Abb.6). Schon heute wird also das Gästeverhalten prinzipiell von den Landschaftsveränderungen beeinflußt: Die Sensiblen "suchen das Weite»! Das Risiko von größeren Nachfrageverschiebungen ist folglich kein Phantom, sondern eine ernstzunehmende Realität.

\section{Wie kann dem Risiko landschaftsbedingter Nachfrageeinbußen begegnet werden? Schlußfolgerungen für die Praxis}

Tourismusbedingte Landschaftsveränderungen werden also von den Touristen negativ erlebt: in Zukunft vielleicht sogar in verstärktem Maße. Dies und vor allem der Nachweis erster Anzeichen von landschaftsbedingten Nachfragereaktionen machen deutlich, daß die Erhaltung der landschaftlichen Schönheit für Tourismusregio-
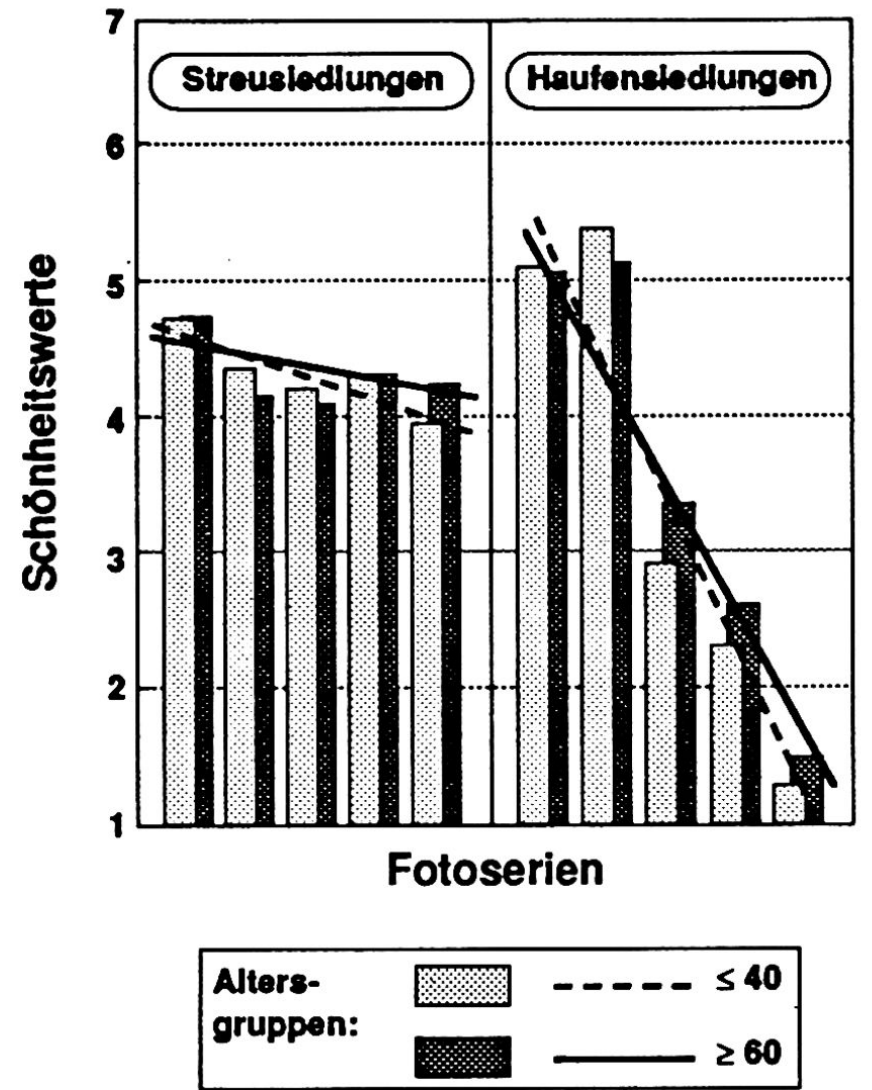

Abb. 6 Die Schönheitswerte der einzelnen Landschaften differenziert nach Gästegruppen mit unterschiedlichem Unterkunftsort in Grindelwald.

nen nicht nur eine ethische Verpflichtung, sondern mittelfristig auch eine ökonomische Notwendigkeit darstellt.

Was nun die Erhaltung der landschaftlichen Schönheit im konkreten Fall bedeutet, kann aus der vorliegenden Studie nicht direkt hergeleitet werden. Möglich sind jedoch einige allgemein gehaltene Vorschläge für künftige Ferienortsplanungen:

- Grundsätzlich ist die traditionelle Kulturlandschaft mit ihren authentischen Siedlungsteilen und landwirtschaftlichen Nutzungsformen zu erhalten.

- Entwicklungen von Haufendörfern hin zu reinen Touristensiedlungen mit städtischem Charakter und ohne lokale Eigenart sollten verhindert werden. Wenn möglich sind bei Neubauten ortstypische Baumaterialien $\mathrm{zu}$ verwenden und die Gebäudedimensionen und -proportionen dem Bestehenden anzupassen.

- Strukturelle Verdichtungen in Streusiedlungsgebieten sind aus ästhetischer Sicht akzeptabel. Es gelten jedoch für die Streusiedlungsentwicklungen dieselben Einschränkungen wie bei den Haufendörfern.

- Weil bei der ästhetischen Beurteilung von Straßen deren Funktion ausschlaggebend ist, sollte darauf geachtet werden, daß in einer Tourismusregion möglichst 
große zusammenhängende Räume vorhanden bleiben, die nicht von Verbindungs- und UmfahrungsstraBen durchschnitten werden.

- Bei der Erstellung neuer touristischer Transportanlagen ist Zurückhaltung geboten. Zurückhaltung heißt dabei «möglichst wenige», weil Bahnen unabhängig von deren Art, Transportkapazität und Linienführung als ästhetisch negativ erlebt werden.

Diese Vorschläge, die entsprechend den lokalen Voraussetzungen weiter konkretisiert werden müßten, beruhen aufden ästhetischen Ansprüchen der Touristen. Die Entscheidung, wie weit diese berücksichtigt werden sollen, liegt bei der einheimischen Bevölkerung, denn diese besitzt die Kompetenz zur Gestaltung ihres Lebensraums seit Jahrhunderten.

\section{Dank}

Mein Dank richtet sich vor allem an Prof. P. Messerli für die Betreuung der Diplomarbeit und an Dr. U. Wiesmann für die wertvollen Anregungen in der Anfangsphase des Projektes. Dr. F. Kienast danke ich für seine Unterstützung bei der Verfassung dieses Artikels.

\section{Anmerkungen}

'Nationales Forschungsprogramm MaB, im Rahmen des Unesco-Programms "Man and Biosphere"

${ }^{2}$ Genaueres kann bei NOHL 1983, NOHL \& NEUMANN 1986, HOISL et al. 1987 und HUNZIKER 1991 nachgelesen werden.

\section{Literatur}

ABT, Th. (1983): Fortschritt ohne Seelenverlust. Bern. BÄTZING, W. (1988): Die Alpen - Naturbearbeitung und Umweltzerstörung. Frankfurt am Main.
FISCHER, D. (1989): Ferienorte auf dem Weg ins Jahr 2000. In: Jahrbuch der Schweizerischen Tourismuswirtschaft, 1988/89.

HOISL, R. et al. (1987): Landschaftsästhetik in der Flurbereinigung. Reihe: Materialien zur Flurbereinigung, Heft 11, München.

HUNZIKER, M.(1991): Landschaftsveränderung - ein Risikofaktor für die touristische Entwicklung des Berggebietes? Diplomarbeit Geogr. Inst. Univ. Bern.

KELLER, P. (1988): Europäische Integration - Touristische Standortbestimmung aus schweizerischer Sicht. In: SFVBulletin, 1988/5, 11-22.

KRIPPENDORF, J. (1986a): Die Landschaftsfresser. Bern KRIPPENDORF, J. (1986b): Alpsegen - Alptraum. Bern.

MAGGI, R. (1983): Entwicklungsmöglichkeiten von Schweizer Wintersportorten. Reihe: St. Galler Beiträge zum Fremdenverkehr und zur Verkehrswirtschaft: Fremdenverkehr, Band 15. Bern.

MESSERLI, P. (1989): Mensch und Natur im alpinen Lebensraum - Risiken, Chancen, Perspektiven. Bern.

NOHL, W. (1983): 30 Thesen einer "anderen" Ästhetik. In: Natur und Landschaft, Nr. 58/1, 18-22.

NOHL, W. (1990): Zur Rolle des Nicht-Sinnlichen in der landschaftsästhetischen Erfahrung: In: Natur und Landschaft, Nr. 65/7+8, 366-370

NOHL, W. \& NEUMANN, K.-D.(1986): Landschaftsbewertung im AlpenparkBerchtesgarden - Umweltpsychologische Untersuchungen zur Landschaftsästhetik. (Deutsche) MABMitteilungen Nr. 23. Bonn.

ROMEISS-STRACKE, F. (1987): Zukünftige Entwicklungsbedingungen für Freizeit und Tourismus. In: ALLGEMEINER DEUTSCHER AUTOMOBILCLUB, Hrsg. (1987): Tourismus auf neuen Wegen - Ein Diskussionsbeitrag des ADAC. München.

STEPHENSON, W. (1953): The Study of Behavior: Q-Technique and its Methodology. Chicago.

TSCHURTSCHENTHALER, P. (1986): Das Landschaftsproblem im Fremdenverkehr - dargestellt anhand der Situation des Alpenraums. Bern.

WIESMAMM, U. (1986): Landschaftliche Schönheit und touristische Nachfrage. In: Jahrbuch der Geographischen Gesellschaft von Bern, Nr. 55, 361-384. 